\title{
GENERICITY OF SIMPLE EIGENVALUES FOR A METRIC GRAPH
}

\author{
LEONID FRIEDLANDER \\ University of Arizona
}

\section{INTRODUCTION}

Let $\Gamma$ be a connected finite graph; by $V$ we denote the set of its vertices, and by $E$ we denote the set of its edges. In a contrast with a combinatorial graph, each edge $e$ is considered to be a line segment of length $l(e)$. Sometimes, it is convenient to treat each edge as a pair of oriented edges; then, on an oriented edge, one defines a coordinate $x_{e}$ that runs from 0 to $l(e)$. If $-e$ is the same edge, with the opposite orientation, then $x_{-e}=l(e)-x_{e}$. If an edge $e$ emanates from a vertex $v$, we will express it by writing $v \prec e$. A good survey of operators on metric graphs and numerous references can be found in $[\mathrm{Ku}]$.

A function $\phi$ on $\Gamma$ is a collection of functions $\phi_{e}(x)$ defined on each edge $e$. We say that it belongs to $L^{2}(\Gamma)$ if each function $\phi_{e}$ belongs to $L^{2}$ on the corresponding edge; then

$$
\|\phi\|^{2}=\sum_{e}\left\|\phi_{e}\right\|^{2} .
$$

The Sobolev space $H^{1}(\Gamma)$ is defined as the space of continuous functions on $\Gamma$ that belong to $H^{1}$ on each edge. The Laplacian on $\Gamma$ is given by the differential expression $-d^{2} / d x_{e}^{2}$ on each edge. To define an operator, one has to specify the domain. For the domain, we take continuous functions that belong to the Sobolev space $H^{2}$ on each edge and that satisfy the Kirchhoff condition

$$
\sum_{e \succ v} \frac{d \phi}{d x_{e}}(v)=0
$$

for every vertex $v$. The operator is induced by the quadratic form

$$
\sum_{e} \int_{e}\left|\frac{d \phi_{e}(x)}{d x_{e}}\right|^{2} d x_{e}
$$

defined on $H^{1}(\Gamma)$. This operator is self-adjoint, and its spectrum consists of eigenvalues

$$
0=\lambda_{1}(\Gamma)<\lambda_{2}(\Gamma) \leq \lambda_{3}(\Gamma) \leq \cdots \nearrow \infty
$$

of finite multiplicity. The eigenvalues are the numbers for which the problem

$$
\frac{d^{2} \phi_{e}}{d x_{e}^{2}}+\lambda \phi_{e}=0
$$


subject to the Kirchhoff conditions (1.1), has a non-trivial solution. For the sake of brevity, we call $\left\{\lambda_{j}(\Gamma)\right\}$ the spectrum of the metric graph $\Gamma$.

The question that we address in this paper is whether generically these eigenvalues are simple. This question has been studied for operators on manifolds (see $[\mathrm{A}]$, $[\mathrm{BU}],[\mathrm{BW}],[\mathrm{U}]$.) Let us formulate the question in more precise terms. We fix the combinatorial structure of $\Gamma$. Then the graph depends on $|E|$ positive parameters $l(e)$. The question is, whether for a generic choice of these parameters the spectrum is simple.

If $\Gamma$ is a polygon then our problem is equivalent to the spectral problem for the Laplacian on the circle of the same circumference, and positive eigenvalues are always double. Therefore, with no additional assumptions, the genericity theorem does not hold. We will show that, for all other graphs, the spectrum of the Laplacian is generically simple.

Suppose that $v$ is a vertex of degree 2 in $\Gamma$. One can remove this vertex and replace two edges $e_{1}$ and $e_{2}$ incident to $v$ by one edge of length $l\left(e_{1}\right)+l\left(e_{2}\right)$. It is easy to see that the spectrum of the resulting graph coincides with the spectrum of $\Gamma$. Therefore, with no lost of generality, one can assume that a graph does not have vertices of degree 2 . In the example from the previous paragraph, all vertices are of degree 2; if all of them are removed, one obtains a circle that we consider to be a metric graph with no vertices.

Theorem. Let $\Gamma$ be a connected metric graph that is different from a circle. Suppose that it does not have vertices of degree 2 . Let $\mathcal{M}$ be the set in the parameter space $\mathbb{R}_{+}^{|E|}$ of metrics, for which all eigenvalues of $\Gamma$ are simple. Then the set $\mathcal{M}$ is residual.

\section{Proof of the Theorem}

First, if $|E|=1$ then we are dealing with the spectrum of the Neumann Laplacian on a segment, which is known to be simple. Therefore, we assume that $|E| \geq 2$.

It is well known that a genericity theorem follows from the fact that a multiple eigenvalue can be split by a small perturbation. Let us formulate it precisely. Suppose that $\lambda$ is a multiple eigenvalue of $\Gamma$ for a certain value of $(l(e))$, and there are no other eigenvalues of $\Gamma$ in an $\epsilon$-neighborhood of $\lambda$. We say that it splits by a small perturbation of parameters if there exists a continuous curve $(l(e, t))$ in the parameter space such that $l(e, 0)=l(e)$, and there are at least two eigenvalues of $\Gamma$ in an $\epsilon / 2$-neighborhood of $\lambda$ for sufficiently small values of $t$ different from 0 .

Lemma. Suppose that the edge lengths $l(e)$ are rationally independent. Then every multiple eigenvalue of $\Gamma$ splits.

First, we deduce the theorem from the lemma. The argument is standard, and we give it for the sake of completeness. Let $\mathcal{M}_{k}$ be the set of all $(l(e))$ for which the first $k$ eigenvalues of $\Gamma$ are simple. The eigenvalues are continuous functions of $l(e)$, so the set $\mathcal{M}_{k}$ is open. We will show that it is dense. Let $(l(e)) \in \mathbb{R}_{+}^{|E|}$. Fix $\delta>0$. We have to show that there exists $\left(l^{\prime}(e)\right) \in \mathcal{M}_{k}$ such that $\left|(l(e))-\left(l^{\prime}(e)\right)\right|<\delta$. Let $\lambda_{j}$ be eigenvalues of $(\Gamma,(l(e)))$ and $\lambda_{j}^{\prime}$ be eigenvalues of $\left(\Gamma,\left(l^{\prime}(e)\right)\right)$. There exist numbers $\epsilon>0$ and $\delta_{1}>0$ such that if $\left.\mid\left(l^{\prime}(e)\right)-l(e)\right) \mid<\delta_{1}$ then

(1) $2 \epsilon$ is smaller than the smallest distance between different eigenvalues $\lambda_{j}$, $j=1, \ldots, k$; 
(2) all eigenvalues $\lambda_{j}^{\prime}$ that are smaller than $\lambda_{k}+\epsilon$ lie in the union of $\left(\lambda_{j}-\epsilon, \lambda_{j}+\epsilon\right)$, $j=1, \ldots, k$

(3) the total multiplicity of the eigenvalues $\lambda_{i}^{\prime}$ that lie in $\left(\lambda_{j}-\epsilon, \lambda_{j}+\epsilon\right), j=$ $1, \ldots, k$, equals the multiplicity of $\lambda_{j}$.

Let $\delta_{2}=\min \left\{\delta, \delta_{1}\right\}$. Choose $(\tilde{l}(e))$ such that $|(\tilde{l}(e))-(l(e))|<\delta_{2} / 2$ and the numbers $\tilde{l}(e)$ are rationally independent. If one out of the first $k$ eigenvalues of $(\Gamma, \tilde{l}(e)))$ is multiple then, by the Lemma, one can make it split and find a point $\left(\tilde{l}^{\prime}(e)\right)$ in a $\delta_{2} / 4$-neighborhood of $(\tilde{l}(e))$ such that the number of different eigenvalues out of the first $k$ eigenvalues of $\left(\Gamma,\left(\tilde{l}^{\prime}(e)\right)\right)$ is bigger than it is for $(\Gamma,(\tilde{l}(e)))$. Because the set of points in $\mathbb{R}^{|E|}$ with rationally independent components is dense, one can assume that the numbers $\tilde{l}^{\prime}(e)$ are rationally independent. If one out of first $k$ eigenvalues of $\left(\Gamma,\left(\tilde{l}^{\prime}(e)\right)\right)$ is still multiple, one repeats the procedure, and, after at most $k$ steps, one gets a point $\left(l^{\prime}(e)\right) \in \mathcal{M}_{k}$ that lies in a $\delta_{1}$-neighborhood of $(l(e))$. We have proved that the set $\mathcal{M}_{k}$ is open, dense. Therefore, the set $\mathcal{M}=\cap_{k} \mathcal{M}_{k}$ is residual.

Proof of the Lemma. Let $\lambda$ be a multiple eigenvalue of $(\Gamma,(l(e)))$. By $M_{\lambda}$ we denote the corresponding space of eigenfunctions. Fix an edge $e_{0}$ and consider a family of metric graphs with $l\left(e_{0}, t\right)=l\left(e_{0}\right)+t$; the lengths of all other edges are kept unchanged. To apply the perturbation theory (e.g., see $[\mathrm{Ka}]$, ) it is convenient to reduce the problem to the problem about a family of operators on a fixed metric graph. To do the job, for small values of $|t|$, we introduce a family of diffeomorphisms $f(t):\left[0, l\left(e_{0}\right)\right] \rightarrow\left[0, l\left(e_{0}\right)+t\right]$ that depends on $t$ smoothly, and such that

(1) $f(0, x)=x$

(2) $f(t, x)=x$ when $0 \leq x \leq l\left(e_{0}\right) / 3$;

(3) $f(t, x)=x+t$ when $2 l\left(e_{0}\right) / 3 \leq x \leq l\left(e_{0}\right)$.

Let $g(t, x)$ be the family of inverse diffeomorphisms. We are using this family, rather than doing a linear rescaling, to keep the Kirchhoff conditions (1.1) unchanged. The Laplacian on $(\Gamma,(l(e, t)))$ is unitary equivalent to the operator $A(t)$ on $(\Gamma,(l(e)))$ given $-d^{2} / d x^{2}$ on the edges $e \neq e_{0}$,

$$
-\left(\frac{\partial g}{\partial x}(t, f(t, x)) \frac{d}{d x}\right)^{2}
$$

on the edge $e_{0}$, and boundary conditions (1.1).

The question of whether a multiple eigenvalue $\lambda$ splits in linear approximation is equivalent to the question of whether the quadratic form $(\dot{A} \phi, \phi)$ is given by a scalar matrix on $M_{\lambda}$. By a dot we denote the $t$-derivative evaluated at $t=0$. Let us compute this quadratic form. First, $\left(\partial^{2} g / \partial x^{2}\right)(0, x)=0$, so

$$
\frac{\partial}{\partial t}\left[\frac{\partial g}{\partial x}(t, f(t, x))\right]_{t=0}=\frac{\partial^{2} g}{\partial x \partial t}(0, x) ;
$$

we denote this function by $h(x)$. The function $h(x)$ vanishes when $x$ is close to 0 or to $l\left(e_{0}\right)$ because of conditions (2) and (3) on $f(t, x)$. We claim that

$$
\int_{0}^{l\left(e_{0}\right)} h(x) d x=-1 .
$$


In fact, if $y=g(t, x)$ and $0 \leq t \leq l\left(e_{0}\right) / 3$ then

$$
l\left(e_{0}\right)-t=\int_{0}^{l\left(e_{0}\right)-t} d y=\int_{0}^{l\left(e_{0}\right)} \frac{\partial g(t, x)}{\partial x} d x .
$$

To get (2.1), one differentiates the last equality in $t$ and sets $t=0$. The operator $\dot{A}$ is given by 0 on all edges $e \neq e_{0}$ and by

$$
-h(x) \frac{d^{2}}{d x^{2}}-\frac{d}{d x} h(x) \frac{d}{d x}
$$

on $e_{0}$. Therefore, for $\phi \in M_{\lambda}$,

$$
(\dot{A} \phi, \phi)=\int_{e_{0}} h(x)\left[\left(\phi^{\prime}(x)\right)^{2}+\lambda \phi(x)^{2}\right] d x .
$$

Here, we have used (1.1), and we have done integration by parts. On $e_{0}$, the function $\phi$ is of the form

$$
\phi(x)=C\left(\phi, e_{0}\right) \sin \left(\sqrt{\lambda} x+s\left(\phi, e_{0}\right)\right)
$$

where $C\left(\phi, e_{0}\right) \geq 0$ and $s\left(\phi, e_{0}\right)$ are constants. Then, (2.1) and (2.2) imply

$$
(\dot{A} \phi, \phi)=-\lambda C\left(\phi, e_{0}\right)^{2} .
$$

From this point, we assume that $\operatorname{dim} M_{\lambda} \geq 2$, and the eigenvalue $\lambda$ does not split. This assumption will lead us to a contradiction. The quadratic form $(\dot{A} \phi, \phi)$ is given by a scalar matrix on $M_{\lambda}$, so

$$
C(\phi, e)=C(\psi, e)
$$

for every edge $e \in E$ and for every pair of eigenfunctions $\phi, \psi \in M_{\lambda}$ such that $\|\phi\|=\|\psi\|=1$. Let $C(e)=C(\phi, e)$ where $\phi \in M_{\lambda}$ and $\|\phi\|=1$.

First, we show that

$$
C(e) \neq 0, \quad e \in E \text {. }
$$

In fact, suppose that $C(e)=0$. Let $v$ be a vertex incident to $e$, and let $e_{1}$ be another edge that has $v$ as its endpoint. Every normalized eigenfunction $\phi \in M_{\lambda}$ vanishes at $v$, so it is of the form $\pm C\left(e_{1}\right) \sin (\sqrt{\lambda} x)$ on $e_{1}$. Let $\phi(t)$ be a smooth one-parameter family of normalized functions from $M_{\lambda}$. All of them are equal to each other on $e_{1}$, so $\phi^{\prime}(t)=0$ on $e_{1}$. However, $\phi^{\prime}(t) \in M_{\lambda}$. Therefore, $C\left(e_{1}\right)=0$. We conclude that if $C(e)=0$ then $C\left(e_{1}\right)=0$ for every edge $e_{1}$ that is adjacent to $e$. Then $C(\tilde{e})=0$ for every edge $\tilde{e} \in E$ because the graph is connected. This is impossible. The contradiction proves (2.6).

Let $\phi$ and $\psi$ be two normalized functions from $M_{\lambda}$ that are orthogonal to each other. Consider the family $\phi(t)=\cos t \phi+\sin t \psi$. Let $v$ be a vertex. if $\phi(v)=$ $\psi(v)=0$ then the functions $\phi$ and $\psi$ coincide on every edge $e$ that is incident to $v$ (see the previous paragraph.) Then, $\phi-\psi=0$ on $e$, and $C(e)=0$, which contradicts (2.6). Therefore, for almost all values of $t$, the function $\phi(t)$ does not 
vanish on all vertices. By redefining $\phi$ and $\psi$, one can assume that the function $\phi$ does not vanish on all vertices.

On an edge $e$

$$
\phi\left(t, x_{e}\right)=C(e) \sin \left(\sqrt{\lambda} x_{e}+s(t, e)\right)
$$

(see (2.3).) Therefore,

$$
\phi_{t}\left(t, x_{e}\right)=s_{t}(t, e) C(e) \cos \left(\sqrt{\lambda} x_{e}+s(t, e)\right), \quad x \in e .
$$

The function $\phi_{t}$ belongs to $M_{\lambda}$, and it is normalized. Its amplitude equals $C(e)$. We conclude that

$$
\frac{d s}{d t}(t, e)= \pm 1
$$

and, therefore,

$$
\phi\left(t, x_{e}\right)=C(e) \sin \left(\sqrt{\lambda} x_{e}+s(e) \pm t\right)
$$

Let $v$ be a vertex, and let $e$ be an edge that emanates from $v$. We differentiate (2.8) with respect to $x_{e}$, and set $t=0, x_{e}=0$ :

$$
\frac{d \psi}{d x_{e}}(v)=-s_{t}(0, e) C(e) \sqrt{\lambda} \sin s(0, e)=-s_{t}(0, e) \sqrt{\lambda} \phi(v) .
$$

From the last equality, from the fact that $\phi(v) \neq 0$, and from (1.1), we conclude

$$
\sum_{e \succ v} s_{t}(0, e)=0 .
$$

Equations (2.9) and (2.12) imply that the degree of each vertex is even. The graph does not have vertices of degree 2 , so the degree of each vertex is at least 4. Moreover, it follows from (2.11) that there exist numbers $\alpha(v)>0$ such that $\left(d \psi / d x_{e}\right)(v)=\alpha(v)$ for half of the edges that emanate from $v$ (we call these edges $v$-positive), and $\left(d \psi / d x_{e}\right)(v)=-\alpha(v)$ for the second half of the edges that emanate from $v$ (we call this edges $v$-negative.) Here, the edges are thought to be oriented, and a loop that emanates from $v$ and terminates at $v$ is counted twice, as $e$ and $-e$.

We call two (oriented) edges, $e$ and $e^{\prime}$, that emanate from the same vertex $v$ neighbors if $\left(d \psi / d x_{e}\right)(v)=-\left(d \psi / d x_{e^{\prime}}\right)(v)$. It follows from (2.10) that then $\left(d \phi(t) / d x_{e}\right)(v)=-\left(d \phi(t) / d x_{e^{\prime}}\right)(v)$ for all values of $t$. We start from a vertex $v_{1}$. Let $e_{1}$ be an edge emanating from $v_{1}$. Denote by $v_{2}$ its terminal vertex. Choose an edge $e_{2}$ that emanates from $v_{2}$ such that $e_{2}$ and $-e_{1}$ are neighbors. Continue the process. We get a sequence of vertices $v_{j}$ and a sequence of edges $e_{j}$ connecting $v_{j}$ with $v_{j+1}$ such that $e_{j+1}$ is a neighbor of $-e_{j}$. At some point, we hit the same vertex twice. We assume that $v_{1}=v_{k+1}$ is the vertex that is repeated the first time; otherwise, we rename that vertex by $v_{1}$. All the vertices $v_{1}, \ldots, v_{k}$ are different. We map the union of edges $e_{j}, j=1, \ldots, k$ onto the interval $[0, l], l=l\left(e_{1}\right)+\cdots+l\left(e_{k}\right)$, by the formula

$$
x_{e_{j}} \mapsto l\left(e_{1}\right)+\cdots+l\left(e_{j-1}\right)+x_{e_{j}} .
$$


By $\phi(t, y)$ we denote the function $\phi(t)$ on $\Gamma$, transplanted to $[0, l]$ according to (2.13). The function $\phi(t, y)$ is continuous, it is differentiable because the adjacent edges are neighbors, and it satisfies the differential equation $\phi_{y y}+\lambda \phi=0$. On the interval $\left[0, l\left(e_{1}\right)\right]$ it is given by $(2.10)$, with $x_{e_{1}}$ replaced by $y$. Therefore,

$$
\phi(t, y)=C\left(e_{1}\right) \sin \left(\sqrt{\lambda} y+s\left(e_{1}\right) \pm t\right)
$$

The endpoints of the interval $[0, l]$ correspond to the same point $v_{1}$ on $\Gamma$, so $\phi(t, 0)=$ $\phi(t, l)$, and

$$
\sin \left(s\left(e_{1}\right) \pm t\right)=\sin \left(l \sqrt{\lambda}+s\left(e_{1}\right) \pm t\right)
$$

for all values of $t$. Hence,

$$
l \sqrt{\lambda} \in 2 \pi \mathbb{Z}_{+}
$$

and

$$
l\left(e_{1}\right)+\cdots+l\left(e_{k}\right)=2 \pi \lambda^{-1 / 2} p, \quad p \in \mathbb{Z}_{+} .
$$

Now, we start the same procedure second time from a vertex $w_{1}$. We use the same rules to construct a sequence of vertices $w_{1}, w_{2}, \ldots$ and a sequence of edges $f_{1}, f_{2}, \ldots$, with one addition: on each step, an edge should be chosen to be different from $e_{1}, \ldots, e_{k}$. Because not more than two edges from $e_{1}, \ldots, e_{k}$ are adjacent to each vertex, the degree of each vertex is at least 4 , and the number of $v$-positive edges equals the number of $v$-negative edges, the process can be continued all the way up to a moment when a repetition in the sequence $w_{1}, w_{2}, \ldots$ arises. Then, we get another cycle $\left(f_{1}, \cdots, f_{m}\right)$ of neighbors, and

$$
l\left(f_{1}\right)+\cdots+l\left(f_{m}\right)=2 \pi \lambda^{-1 / 2} q, \quad q \in \mathbb{Z}_{+} .
$$

The edges $e_{j}$ are different from the edges $f_{i}$, so (2.14) and (2.15) contradict to the assumption of the lemma that the numbers $l(e)$ are rationally independent.

\section{REFERENCES}

[A] J. Albert, Genericity of simple eigenvalues for elliptic PDE's, Proc. Amer. Math. Soc. 48 (1975), 413-418.

[BU] S. Bando, H. Urakawa, Generic properties of eigenvalues of the Laplacian for compact Riemannian manifolds, Tôhoku Math. J. 35 (1983), 155-172.

[BW] D. Bleecker, L. Wilson, Splitting the spectrum of a Riemannian manifold, Siam J. Math. Analysis 11 (1980), 813-818.

[Ka] T. Kato, Perturbation theory for linear operators, Springer-Verlag, Berlin-Heidelberg-New York, 1966.

[Ku] P. Kuchment, Quantum graphs: I. Some basic structures, Waves Random Media 14 (2004), S107-S128.

[U] K. Uhlenbeck, Generic properties of eigenfunctions, Amer. J. Math. 98 (1976), 1059-1078.

Department of Mathematics University of Arizona Tucson, AZ 85721

E-mail address: friedlan@math.arizona.edu 